\title{
META-HEURISTIC APPROACH FOR HIGH-DEMAND FACILITY LOCATIONS CONSIDERING TRAFFIC CONGESTION AND GREENHOUSE GAS EMISSION
}

\author{
Taesung HWANG ${ }^{\mathrm{a}}$, Minho LEE ${ }^{\mathrm{b}}$, Chungwon $\mathrm{LEE}^{\mathrm{c}}$, Seungmo KANG \\ a Asia Pacific School of Logistics and Graduate School of Logistics, Inha University, \\ 100 Inha-ro, Nam-gu, Incheon, 402-751, Republic of Korea \\ ${ }^{b}$ School of Civil, Environmental, and Architectural Engineering, Korea University, \\ 145 Anam-ro, Sungbuk-gu, Seoul, 136-713, Republic of Korea \\ 'Department of Civil and Environmental Engineering, Seoul National University, \\ 599 Gwanak-ro, Gwanak-gu, Seoul 151-744 Republic of Korea
}

Submitted 25 Nov. 2015; accepted 02 Jun. 2016

\begin{abstract}
Large facilities in urban areas, such as storage facilities, distribution centers, schools, department stores, or public service centers, typically generate high volumes of accessing traffic, causing congestion and becoming major sources of greenhouse gas (GHG) emission. In conventional facility-location models, only facility construction costs and fixed transportation costs connecting customers and facilities are included, without consideration of traffic congestion and the subsequent GHG emission costs. This study proposes methods to find high-demand facility locations with incorporation of the traffic congestion and GHG emission costs incurred by both existing roadway traffic and facility users into the total cost. Tabu search and memetic algorithms were developed and tested with a conventional genetic algorithm in a variety of networks to solve the proposed mathematical model. A case study to determine the total number and locations of community service centers under multiple scenarios in Incheon City is then presented. The results demonstrate that the proposed approach can significantly reduce both the transportation and GHG emission costs compared to the conventional facility-location model. This effort will be useful for decision makers and transportation planners in the analysis of network-wise impacts of traffic congestion and vehicle emission when deciding the locations of high demand facilities in urban areas.
\end{abstract}

Keywords: vehicle GHG emission, logistics systems planning, facility location model, traffic congestion, meta-heuristic algorithm, tabu search algorithm, memetic algorithm, genetic algorithm.

\section{Introduction}

Rapid growth in passenger and freight transportation had significantly affected environmental systems. According to the U.S. EPA (2013), total greenhouse gas (GHG) emissions in the U.S. from the transportation sector increased by about $18 \%$ from 1990 to 2011 . During this period, road transport was responsible for more than $70 \%$ of the total emissions. In South Korea, the environmental cost of vehicle emissions in 2010 was estimated to be around $7 \%$ of the total social cost, including congestion, accidents, air pollution, and noise, which is greater than the total cost of traffic accidents (Statistics Korea 2014). Environmental and political efforts have been made by many governments and authorities in an attempt to improve air quality and social welfare (Jang et al. 2010; Lee, J. K., Lee, S. 2010).
For example, the Air Resource Board of the California Environmental Protection Agency (2014) has been conducting programs to reduce emissions in the state from mobile sources, including passenger cars and heavy-duty trucks. In similar jurisdictions, the environmental impacts of transportation systems have become significant problems, especially in congested urban roadway networks. Improvements are also needed for reduction of the vehicle emissions in supply-chain systems.

Many studies of supply-chain systems have explored the environmental impacts of vehicle emissions. Figliozzi (2011b) studied the vehicle routing problem (VRP), in which minimization of emissions is incorporated into the objective. That study demonstrated why vehicle emissions should be considered in the supply-chain network of private companies. Wang et al. (2011) studied a supply-chain

Corresponding author: Seungmo Kang

E-mail: s_kang@korea.ac.kr 
network design problem wherein environmental impacts were considered in a multi-objective optimization model. Srivastava (2007) conducted an extensive review of green supply-chain problems. The impacts of vehicle emissions also have been incorporated into some conventional facility-location problems. For example, Diabat and SimchiLevi (2010) studied a multi-commodity facility-location problem with a carbon-emission constraint. Harris et al. (2009) proposed an uncapacitated facility-location problem in the form of multi-objective models, through the examination of two objectives, including economic cost versus environmental impact, and three objectives, including economic cost, environmental impact, and uncovered demand. Harris et al. (2011) investigated the capacitated facility-location problem in which the financial cost and the $\mathrm{CO}_{2}$ emission cost were considered as a multi-objective function. Such studies provide insight into how environmental considerations can be incorporated into the classical fixed charge location problem (FCLP). However, they do not carefully address the congestion effects caused by facility users, especially in urban environments with high traffic volumes. In addition, the environmental impacts of re-routing background traffic, which represents general roadway users unrelated to the facilities, have seldom been considered.

Conventional facility-location problems applied to supply-chain design often consider facility investment and operation costs, as well as the fixed transportation costs of connecting customers with the facilities. FCLP, an important facility-location model, has been used extensively, both in practice and in academic studies (Nozick 2001). It has also been extended into capacitated and uncapacitated versions (Erlenkotter 1978; Aikens 1985). Pirkul and Jayaraman (1998) investigated a multi-commodity multiplant capacitated FCLP. Aksen and Aras (2012) investigated a bi-level FCLP in which vulnerability of the system to potential terrorist attacks was considered. Heuristic algorithms have been proposed to solve these NP-hard problems. Sridharan (1995) reviewed the capacitated FCLP and a set of solution algorithms, including heuristics and exact methods. Cornuejols et al. (1991) compared several heuristics to solve capacitated FCLP. Lagrangean Relaxationbased heuristics were investigated by Beasley (1993) and Tragantalerngsak et al. (1997), and were further extended to the FCLP under random disruptions (Lim et al. 2010). Meta-heuristic approaches also have been developed, such as tabu search (Al-Sultan, Al-Fawzan 1999; Prins 2007), ant colony optimization (Venables, Moscardini 2006), and combinations of construction heuristics and a local search algorithm (Sörensen et al. 2012) for a capacitated FCLP. Jaramillo et al. (2002) reviewed the use of genetic algorithms in general facility-location problems including FCLP, which was followed by the studies of Raj and Rajendran (2012) and Xie and Jia (2012). Arostegui et al.
(2006) compared tabu search, simulated annealing, and genetic algorithms for a capacitated, multi-period, multicommodity version of FCLP. As outlined above, extensive studies have been conducted and various solutions have been proposed for FCLP and its variations. However, in most urban environments where the existing traffic is at or near capacity, new facilities can generate additional traffic which may result in huge impacts on the area-wide congestion, and consequently, on the total vehicle emissions (Shukla, Alam 2010). Once new facilities start operating and the traffic increases, conventional facility-location models with fixed transportation costs may not be able to properly address the problem.

This paper aimed to fill these gaps by presenting a comprehensive mathematical model which considers (i) network-wise congestion caused by both re-routing of existing traffic and assignment of facility users on the given networks, and (ii) the environmental impact of vehicle movements (i.e., both existing traffic and facility users) associated with facility locations. When high-demand facilities are built in urban areas, the facility users generate additional trips which may increase traffic congestion not only around the facilities, but also in the whole transportation network of the larger area. This congestion can also alter the routes of other general drivers, finally causing the traffic pattern to converge to another equilibrium state. The amount of $\mathrm{CO}_{2}$ emissions resulting from this updated traffic should be explicitly considered. A study by Bai et al. (2011) explored the interactions between a facility-location problem and a transportation-planning problem, in which the facility locations were iteratively updated considering the roadway congestion patterns associated with shipment origins and destinations. However, the existing background traffic on the transportation network was assumed to be fixed and not affected by facility-location decisions or the resulting traffic congestion pattern. A few studies considered the re-routing of background traffic in supply-chain design problems, but such re-routing has not been applied in a real-world case study (Hajibabai, Ouyang 2013), or was only tested on a small-sized network (Hajibabai et al. 2014). Moreover, none of the models consider vehicle emission cost, which becomes more significant during heavy network congestion. In this study, we explicitly incorporate (i) background traffic movement to minimize congestion, and (ii) emission costs caused by general roadway users as well as facility users, into the objective function. The proposed model and solution algorithm are applied to a large-scale network to find the near-optimal number and locations of community service centers in the city of Incheon, South Korea.

The exposition of this paper is as follows. Section 1 presents the notations used in the model and the mathematical formulation. The proposed solution algorithms, including tabu search and a memetic algorithm, are 
introduced in Section 2. Section 3 focuses on a set of case studies, and provides a discussion of the scenario analyses. The last section concludes the study and suggests points for future research.

\section{Model formulation}

In this section, a bi-level mixed-integer program which combines the fixed-charge facility-location model and the route-choice model is presented for consideration of all travelers on the network, including background traffic and facility users. The method synchronously addresses facility location, traffic congestion induced by high-demand facilities, and the consequent vehicle emissions. An upperlevel model was formulated to find the optimal number and locations of facilities which allow minimization of the sum of facility construction cost, transportation cost, and emission cost. A lower-level model then addresses the traffic assignment problem of both facility demand and background traffic, in which the user equilibrium principle (Wardrop 1952) was adopted as the rule for route choice. Therefore, it was assumed that each traveler would select the path with the shortest travel time between his/ her origin and destination. This leads to the equilibrium state, in which no traveler can reduce travel time by changing paths.

The roadway network is represented by a directed graph $D(V, A)$, where: $V$ is the set of nodes; $A$ is the set of directed links. Let $J \subseteq V$ be a set of candidate facility locations, and $e_{j}$ be a fixed investment for the construction and operation of the facility, $j \in J$. Any facility $j \in J$ is associated with its maximum capacity, $C_{j}$, an upper-bound to the total customer demand that can be assigned to the facility. The binary decision variable $Y_{j}, \forall j \in J$ denotes whether or not the candidate location $j \in J$ is selected for facility construction; $Y_{j}=1$ if a facility is built at candidate location $j$, or 0 otherwise. Let $I^{b} \subseteq V$ and $K \subseteq V$ respectively denote the set of origins and destinations of the background traffic, representing general roadway users. Let $I^{d} \subseteq V$ be the set of origins of facility demand and $h_{i}$ be the facility demand at origin $i \in I^{d}$. The total facility demand in the network can be described as $\sum_{i \in I^{d}} h_{i}$.

Assuming that the number of facilities and their locations are provided, facility demand at each origin node $i \in I^{d}$ should be properly assigned to the facilities accessible through a given roadway network. A virtual sink node $S$ was proposed to represent an imaginary single destination, and a set of dummy links was defined as $Z$ to connect the candidate location $j \in J$ and $S$ using the dummy link $z_{j} \in Z$ if a facility is constructed at node $j$. Figure 1 shows a detailed illustration of the proposed method. Facility demand $h_{i}$ in each origin node $i \in I^{d}$

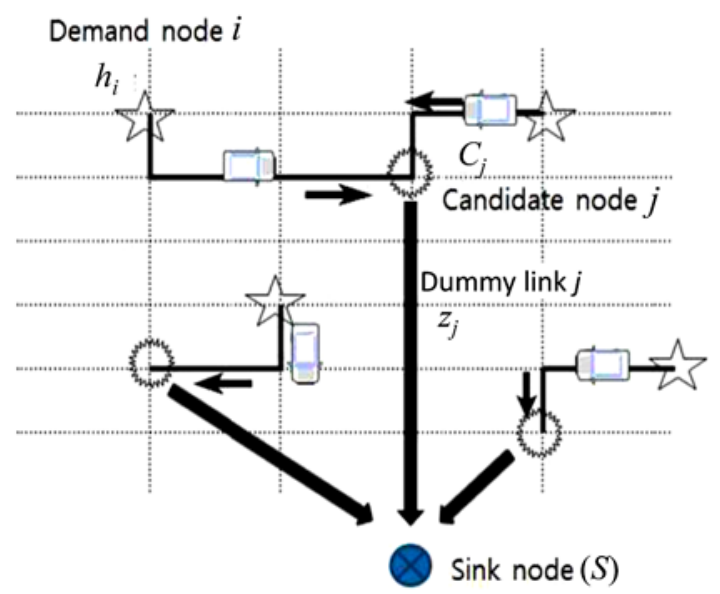

Fig. 1. Transportation network with imaginary sink node and dummy links (revised from Bai et al. 2011)

is assigned to the imaginary single destination $S$ through the intermediate destinations (i.e., constructed facilities). The throughput of each constructed facility is allowed to be up to $C_{j}, \forall j \in J$. With this imaginary single destination, a new origin-destination table does not need to be built for the facility users. The users are assigned to the nearest facility allowed by the capacity, with updated travel time.

Let $x_{a}$ be the total vehicle traffic on the network link, $a \in A$, which includes background traffic flow and facility demand flow on the given link. Let $q^{i, k}$ be the total background traffic flow from origin $i \in I^{b}$ to destination $k \in K$, and let $b_{m}^{i, k}$ be a part of $q^{i, k}$ on any path $m \in M^{i, k}$, where: $M^{i, k}$ is the set of possible paths connecting the given origin-destination pair. The facility demand flow from origin $i \in I^{d}$ to the virtual sink node $S$ on any path $l \in L^{i}$ is represented by $f_{l}^{i}$, where: $L^{i}$ is the set of possible paths connecting the given facility demand node and the imaginary sink node $S$. Then, the total vehicle flow on the link $a \in A$ can be formulated as $x_{a}=\sum_{i \in I^{d}} \sum_{l \in L^{i}} f_{l}^{i} \delta_{a, l}^{i}+\sum_{i \in I^{b}} \sum_{k \in K} \sum_{m \in M^{i, k}} b_{m}^{i, k} \rho_{a, m}^{i, k}$, where: $\delta_{a, l}^{i}=1$ if link $a$ is included as part of path $l$, or 0 otherwise. In addition, $\rho_{a, m}^{i, k}=1$ if link $a$ is included in path $m$, or 0 otherwise. Link travel time (i.e., link cost) can generally be represented as a function of the total vehicle volume on the link, e.g., through the BPR (US Bureau of Public Roads) link cost function (Sheffi 1985). Although vehicle $\mathrm{CO}_{2}$ emissions are affected by a number of factors (e.g., ambient temperature, engine temperature at start up, etc.), vehicle speed, which is uniquely determined by the total vehicle volume on the link from a proper link cost function (Sheffi 1985), is known to be the dominant factor (Figliozzi 2011a). Therefore, in this study, both link travel time and link specific $\mathrm{CO}_{2}$ emission factors were treated as functions of the total vehicle volume on the link, and can 
be respectively described as $t_{a}\left(x_{a}\right)$ and $E_{a}\left(x_{a}\right), \forall a \in A$. Let $v_{j}$ denote the total assigned demand flow for the constructed facility at node $j \in J$, which can be represented as $v_{j}=\sum_{i \in I^{d}} \sum_{l \in L^{i}} f_{l}^{i} \theta_{j, l}^{i}$ where: $\theta_{j, l}^{i}=1$ if the demand flow $f_{l}^{i}$ passes through the constructed facility at node $j$ to reach the sink node $S$, or 0 otherwise. Note that the assigned demand flow for each constructed facility also describes the throughput of each constructed facility, whose upperbound is set as $C_{j}, \forall j \in J$.

Using the inputs and decision variables described above, our model can be formulated into the following bilevel mixed-integer program:

Minimize

$$
\sum_{j \in J} e_{j} Y_{j}+\sum_{a \in A}\left\{\alpha x_{a} t_{a}\left(x_{a}\right)+\beta x_{a} E_{a}\left(x_{a}\right)\right\}
$$

subject to

$$
\begin{gathered}
h_{i}=\sum_{l \in L^{i}} f_{l}^{i}, \forall i \in I^{d} ; \\
\sum_{i \in I^{d}} h_{i} \leq \sum_{j \in J} C_{j} Y_{j} ; \\
Y_{j}=\{0,1\}, \forall j \in J,
\end{gathered}
$$

where: $x_{a}$ is a solution to the following traffic assignment problem under a user equilibrium objective:

Minimize

subject to

$$
\sum_{a \in A} \int_{0}^{x_{a}} t_{a}(\omega) d \omega
$$

$$
\begin{gathered}
x_{a}=\sum_{i \in I^{d}} \sum_{l \in L^{i}} f_{l}^{i} \delta_{a, l}^{i}+\sum_{i \in I^{b}} \sum_{k \in K} \sum_{m \in M^{i, k}} b_{m}^{i, k} \rho_{a, m}^{i, k}, \forall a \in A ; \\
q^{i, k}=\sum_{m \in M^{i, k}} b_{m}^{i, k}, \forall i \in I^{b}, k \in K ; \\
v_{j}=\sum_{i \in I^{d}} \sum_{l \in L^{i}} f_{l}^{i} \theta_{j, l}^{i}, \forall j \in J ; \\
v_{j} \leq C_{j} Y_{j}, \forall j \in J ; \\
f_{l}^{i} \geq 0, \forall i \in I^{d}, \quad l \in L^{i} ; \\
b_{m}^{i, k} \geq 0, \forall i \in I^{b}, \quad k \in K, \quad m \in M^{i, k} .
\end{gathered}
$$

The objective function (1) of the upper-level problem minimizes the sum of the facility building cost, total travel time and the related emission costs of both background traffic and facility users. Parameters $\alpha$ and $\beta$ respectively convert travel time in hours and amount of $\mathrm{CO}_{2}$ emission in grams into monetary values. Constraint (2) ensures that the total facility demand at any origin node is the same as the sum of the traffic flow out of that demand node. Constraint (3) ensures that the total facility demand in the network is less than or equal to the sum of the constructed facility capacity. Constraint (4) defines the binary variables.
In the case of the second-level problem, objective function (5) minimizes the sum of the link cost functions across all network links, integrated with respect to the link flow. Constraint (6) defines the total traffic flow on link $a \in A$ as the sum of the facility demand flow and the background traffic flow. Constraint (7) ensures that the total background traffic volume for each origin-destination pair is equal to the sum of the background traffic volume on any possible routes connecting that origindestination pair. Constraint (8) specifies that the assigned demand flow for each constructed facility is the same as the sum of the demand flow passing through the facility to reach the sink node. Constraint (9) ensures that the total assigned demand flow for any constructed facility is less than or equal to the capacity of that facility, and that no facility demand is assigned at candidate nodes where facilities are not constructed. Constraints (10) and (11) specify the non-negative variables. Note that facility demand and background traffic are interdependent, and are thus assigned together by user equilibrium formulas.

The suggested modeling framework proposes a bidirectional relationship between the facility location model (i.e., the upper-level problem) and the traffic assignment model of both background traffic and facility users (i.e., the lower-level problem), considering vehicle emission. Given a feasible solution (i.e., number and location of facilities to be built) from the upper-level problem, general roadway users as well as facility users need to decide routes to their destinations which can avoid congestion. On the other hand, the updated congestion patterns in the network from the lower-level problem in turn influences the decision on facility location in the upper-level problem. As such, the solution from one side alters the solution from the other side iteratively until convergence of the upper-level total cost. In this process, the model explicitly considers the re-routing of general roadway users, along with the vehicle emissions from all traffic in the network.

\section{Solution algorithm}

The proposed mathematical model (1)-(11) is a mixedinteger-program problem with nonlinear functions, which makes it difficult to solve with exact optimality. Consequently, we suggest the use of meta-heuristic algorithms to allow near-optimal solutions to be obtained efficiently in a reasonable amount of time. These include tabu search and memetic algorithm.

\subsection{Tabu search}

Tabu search uses a local search heuristic from an initial solution, or the current best solution, to iteratively search for the best available neighborhood solution until the stop criterion is satisfied. Compared to genetic algorithms, this 
approach has been shown to provide better solutions with shorter computation times in a number of optimization problems (Arostegui et al. 2006). The detailed procedures are described below (Brownlee 2013):

Step 0: Initialization. A solution vector $\mathbf{Y}$ represents a set of binary integer variables $Y_{j}=\{0,1\}, \forall j \in J$ with the length $|J|$, which indicate the locations of facilities. A drop heuristic (Daskin 1995) has been applied to generate an initial solution. The initial objective function value $\mu$ is obtained based on $\mathbf{Y}$. Let $r=0$ count the number of failures in updating the solution vector, and define $N$, a significantly large number, to represent the maximum value that $r$ can take. Define a Tabu list $R=\phi$ whose maximum size, $|R|$, is the same as the total number of candidate locations, $|J|$, to enhance the performance of the algorithm by forbidding moves to solutions that have been visited previously.

Step 1: Define $H$ as a set of neighboring solutions from the current best solution, which is initially $\phi$. To generate the neighboring solutions, we adopted a neighborhood search heuristic in which the binary values in each candidate location in a vector $\mathbf{Y}$ are changed oneby-one. Among the neighboring solutions in the set $H$, select the one that leads to the highest improvement in the value of the objective function. The objective function value is calculated by the user equilibrium assignment result with the convex combinations algorithm (Sheffi 1985). The travel time and emission cost come from each link volume and link speed. Facility costs are obtained from $\mathbf{Y}$. If the selected solution contains elements in the Tabu list, select the next best neighboring solution and repeat the checking procedure with the Tabu list; otherwise, let $\mathbf{Y}^{\prime}$ be the selected solution representing the best available neighboring solution from the set $H$, and let the new objective function value based on $\mathbf{Y}^{\prime}$ be $\mu^{\prime}$.

Step 2: If $\mu^{\prime}$ is better (i.e., lower) than $\mu$, the solution $\mathbf{Y}^{\prime}$ is set as $\mathbf{Y}$ (i.e., the current best solution) and the features of $\mathbf{Y}^{\prime}$ are included into the Tabu list as the last element (at this point, if $|R|>|J|$, eliminate the first element in the Tabu list); otherwise, update $r \leftarrow r+1$.

Step 3: If any termination criterion is met (e.g., $r$ is greater than $N$, solution time becomes greater than the time limit, or the objective value converges), terminate the algorithm with the solution $\mathbf{Y}$; otherwise, go back to Step 1.

\subsection{Memetic algorithm}

Memetic algorithms were developed to improve the performance of traditional genetic algorithms. The basic procedures are very similar to those of the genetic algorithm, but a neighborhood search method is incorporated into the survival-of-the-fittest rule. The following steps describe the memetic algorithm in detail (Brownlee 2013):
Step 0: Initialization. Parameters including the population size and stop criteria are defined. A chromosome is represented by a solution vector $\mathbf{Y}$, which includes a set of binary integer variables $Y_{j}=\{0,1\}, \forall j \in J$ in each cell. Chromosomes are randomly generated to form the first population, in which elitism is applied to achieve faster convergence of the objective value and to carry over superior genes from the current generation to the next generation. An initial solution is created by applying the drop heuristic (Daskin 1995).

Step 1: Evaluate the fitness value of each chromosome - the percentage ratio of the inverse of the objective value associated with the chromosome to the total sum of the inverses of the objective values associated with all chromosomes. Given a chromosome, the calculation of the objective value is equivalent to that of the tabu search algorithm. Next, randomly select two chromosomes from the parent chromosome pool and compare their fitness values. The one with the higher fitness value is selected via the tournament selection (Goldberg 1989) technique, and its genes are then carried over to the next generation.

Step 2: A one point crossover is conducted, in which two identical points are arbitrarily selected from two different chromosomes (i.e., both cross points represent decision variables for the same candidate location) randomly chosen from the population. The values in the selected genes are exchanged to create a new pair of chromosomes. Mutation is then applied, in which different mutation probabilities are assigned to different cells in the chromosome and a random number between 0 and 1 is generated. If the probability associated with each cell is larger than the random number, the binary variable assigned to the cell is flipped; otherwise, the binary variable is unchanged.

Step 3: The neighborhood search is applied for each chromosome. As in the tabu search algorithm, the binary value of each cell is changed one-by-one and the original chromosome is replaced with the best neighboring solution. For greater improvement, this process can be repeated.

Step 4: If any termination criteria are satisfied (e.g., solution time reaches the limit or the objective value converges), terminate the algorithm with the solution Y; otherwise, go back to Step 1.

\section{Case study}

The proposed methods were coded in $\mathrm{VC}++$ and tested on a personal computer with a $2.93 \mathrm{GHZ} \mathrm{CPU} \mathrm{and} 8 \mathrm{~GB}$ memory to find the total number and locations of community service centers in various transportation networks. This test was conducted to compare the performances of different algorithms in solving the same problems. Note that a conventional genetic algorithm was also applied. Community service centers are large buildings which offer various 
a)

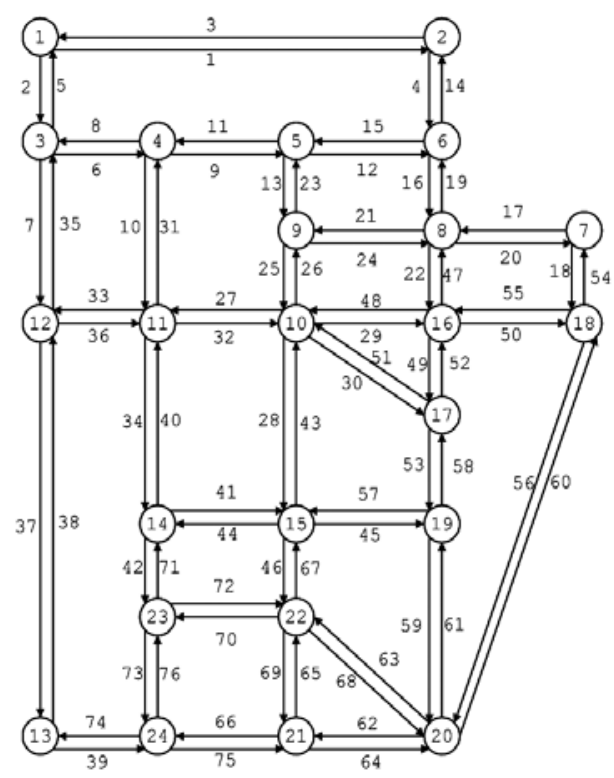

b)

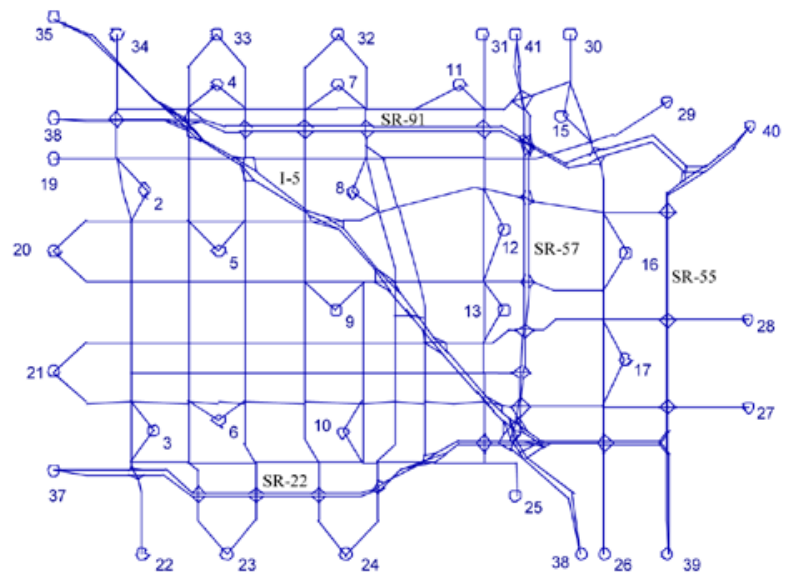

c)

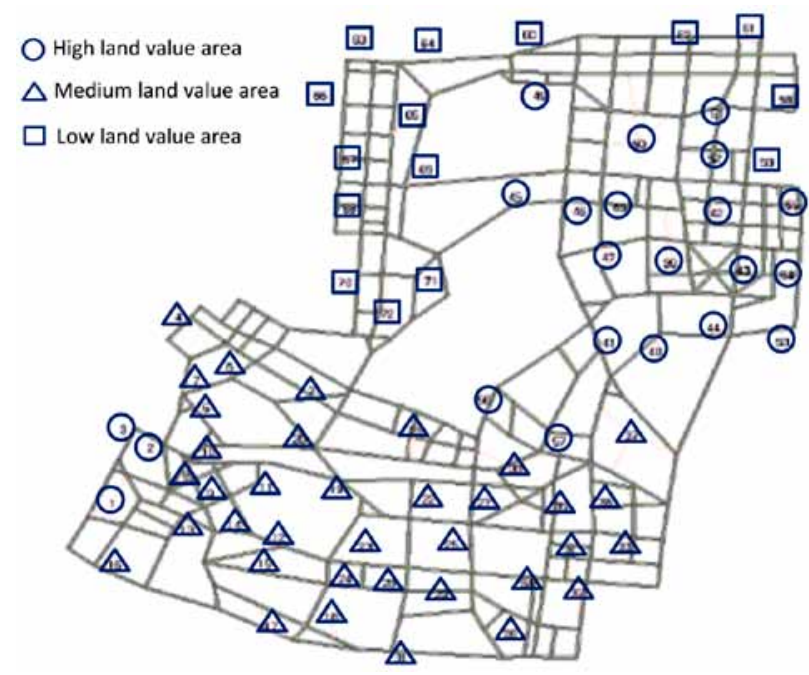

Fig. 2. Test networks, 24-node and 76-link Sioux Falls Network (Bar-Gera 2009) (a), 416-node and 914-link Anaheim Network (Bar-Gera 2009) (b), and 408-node and 1,282-link Incheon Network (KTDB 2014) (c) public services, such as local administrative services, postal services, healthcare services, auditoriums, libraries, recreational services, etc. The modeling framework presented in this paper is a general framework which can be applied to a wide range of entities in supply chain systems, for example, to warehouses, distribution centers, and factories.

Here, we considered small, medium, and large-scale test networks: the 24-node and 76-link Sioux Falls network (Bar-Gera 2009) with 24 candidate locations, shown in Figure 2(a); the 416-node and 914-link Anaheim network (Bar-Gera 2009) with 38 candidate locations, shown in Figure 2(b); and the 408-node and 1,282-link Incheon network (KTDB 2014) with 72 candidate locations, shown in Figure 2(c). The capacity of each facility was assumed to be 5,000 vehicles/day. Facility building costs were converted into daily values with a 20 -year planning horizon. Note that the facility building costs in the Incheon network were categorized into three levels depending on the land values of the candidate locations, including areas with high, medium, and low land value.

All algorithms were terminated when the solution time reached $200 \mathrm{sec}$ for the Sioux-Falls network, $400 \mathrm{sec}$ for the Anaheim network, and 15,000 sec for the Incheon network. The facility demands for all origin nodes (i.e., $h_{i}, \forall i \in I^{d}$ ) were assumed to be given. The BPR link cost function (Sheffi 1985) was used to measure the travel time in hours on each link $a \in A$, as follows:

$$
t_{a}\left(x_{a}\right)=t_{a}^{f}\left[1+0.15\left(\frac{x_{a}}{Q_{a}}\right)^{4}\right], \forall a \in A,
$$

where: $t_{a}^{f}$ is the link travel time in hours without congestion (i.e., free flow travel time); $x_{a}$ is the total traffic volume of the facility demand and background traffic in number of vehicles/hour; $Q_{a}$ is the link capacity in number of vehicles/hour. Note that a modified Davidson's function (Davidson 1966; Kim 2012) was applied to the dummy link $z_{j} \in Z$ to ensure the capacity constraints of the constructed facilities, i.e., equation (9), as follows:

if $v_{j} \leq C_{j}, \quad t_{z_{j}}\left(v_{j}\right)=0.001 \frac{1.1 C_{j}-(1-\gamma) v_{j}}{1.1 C_{j}-v_{j}} ;$

otherwise, $\quad t_{z_{j}}\left(v_{j}\right)=0.001 \frac{1.1-(1-\gamma)}{0.1 \gamma}\left(\frac{v_{j}}{C_{j}}\right)^{2}$,

where: the parameter $\gamma$ was set to 0.0001 to make the dummy link travel time significantly small when $v_{j} \leq C_{j}$. Once $v_{j}$ becomes larger than the given capacity, $C_{j}$, the dummy link travel time increases greatly, using equation (14), which forces the assigned traffic demand on the constructed facility $j$ to be less than or equal to the capacity. All kinds of vehicle volumes including trucks, buses, and $\mathrm{RV}$ s are converted into passenger car volume in equations (12)-(14). Note that the simple approach of using 
passenger car volume might affect the results in real-world case study. This issue could be resolved by applying proper values of passenger car equivalents (PCE) to other type of vehicle (TRB 2000). The $\mathrm{CO}_{2}$ emission factor associated with each link $a, \forall a \in A$ was calculated using the method from Hickman (1999), as follows:

$$
\begin{aligned}
& E_{a}\left(x_{a}\right)=d_{a}\left[u_{0}+u_{1}\left(\frac{d_{a}}{t_{a}\left(x_{a}\right)}\right)+u_{2}\left(\frac{d_{a}}{t_{a}\left(x_{a}\right)}\right)^{2}+\right. \\
& u_{3}\left(\frac{d_{a}}{t_{a}\left(x_{a}\right)}\right)^{3}+u_{4}\left(\frac{d_{a}}{t_{a}\left(x_{a}\right)}\right)^{-1}+ \\
& \left.u_{5}\left(\frac{d_{a}}{t_{a}\left(x_{a}\right)}\right)^{-2}+u_{6}\left(\frac{d_{a}}{t_{a}\left(x_{a}\right)}\right)^{-3}\right],
\end{aligned}
$$

where: link distance is represented by $d_{a}$ (in $\mathrm{km}$ ) and the coefficients are given as $u_{0}=429.51, u_{1}=-7.8227, u_{2}=$ 0.0617, and $u_{3}=u_{4}=u_{5}=u_{6}=0$ (Hickman 1999). In Equation (1), the parameter $\alpha$ was assumed to be 7.5 (\$/ vehicle-hour) (MLTM 2011) to convert time in hours into the monetary values (i.e., dollars). The cost of a ton of $\mathrm{CO}_{2}$ emission, $\beta$, was set to $150\left(\$ / \mathrm{tCO}_{2}\right)$, as suggested by literature in various fields (API 1998; Parry, Toman 2001; Jaccard 2005; RSCEP 2010; MLTM 2011; NRT 2012). Note that MLTM (2011) provides legal guidelines of basic parameters used for evaluating government-funded transportation facility projects in Republic of Korea.
Table 1 provides a summary of the computation results of the three test networks. Columns (a)-(c) respectively show the objective values and solution time of each network when the algorithm reached the lowest objective value. For the Sioux-Falls network, the three methods yielded the same total cost due to the small size of the network. However, the time to achieve this value was the lowest using the tabu search. For the Anaheim and Incheon networks, the tabu search generated the best solution, followed by the memetic algorithm. The genetic algorithm yielded the worst solution among all methods.

To investigate how the consideration of traffic congestion affects total system-wide cost, two designs were proposed for comparison. The first is the conventional fixed-charge facility-location problem in which every traffic demand is assigned to the shortest-distance path while ignoring congestion effects, and thus, vehicle emission is not included in the objective function. The second is our proposed model in which network congestion was considered in assigning the traffic demand resulting from both facility users and general roadway users, for which the GHG emission cost is included in the objective function. The conventional problem of the first design was solved by the Lagrangian relaxation algorithm (Daskin 1995 ) with less than a $0.1 \%$ gap. Tabu search was applied in the second design since it demonstrated the best performance, as shown in Table 1 . The results obtained from

\begin{tabular}{|c|c|c|c|c|}
\hline & & (a) Tabu search & (b) Memetic algorithm & (c) Genetic algorithm \\
\hline \multirow{2}{*}{$\begin{array}{l}\text { Sioux-Falls } \\
\text { network }\end{array}$} & Objective value (\$) & 2.533.E+05 & 2.533.E +05 & 2.533.E+05 \\
\hline & Solution time (sec) & 16.7 & 109 & 157 \\
\hline \multirow{2}{*}{ Anaheim network } & Objective value (\$) & 2.126.E+05 & 2.266.E+05 & 2.276.E+05 \\
\hline & Solution time (sec) & 257 & 359.5 & 74 \\
\hline \multirow{2}{*}{ Incheon network } & Objective value (\$) & 2.611.E+05 & 2.644.E+05 & 2.647.E+05 \\
\hline & Solution time (sec) & 11,613 & 7,416 & 563 \\
\hline
\end{tabular}
each test network in Figure 2 are summarized in Table 2.

Table 1. Computation results for numerical experiments

\begin{tabular}{|c|c|c|c|c|c|}
\hline Network & Design & $\begin{array}{c}(\mathrm{a}) \\
\text { Total cost }(\$)\end{array}$ & $\begin{array}{c}\text { (b) } \\
\text { Facility cost }(\$)\end{array}$ & $\begin{array}{c}\text { (c) Transportation } \\
\text { cost }(\$)\end{array}$ & $\begin{array}{c}\text { (d) } \mathrm{CO}_{2} \text { emission } \\
\text { cost }(\$)\end{array}$ \\
\hline \multirow{3}{*}{ Sioux-Falls } & Ignoring congestion & 254,555 & 2,560 & 187,133 & 64,862 \\
\hline & Congestion and emission included & 253,315 & 3,200 & 187,059 & 63,056 \\
\hline & $\%$ difference & 0.31 & -20.00 & 0.04 & 2.86 \\
\hline \multirow{3}{*}{ Anaheim } & Ignoring congestion & 212,840 & 2,560 & 157,941 & 52,339 \\
\hline & Congestion and emission included & 212,630 & 3,840 & 156,990 & 51,800 \\
\hline & $\%$ difference & 0.10 & -33.33 & 0.61 & 1.04 \\
\hline \multirow{3}{*}{ Incheon } & Ignoring congestion & 286,500 & 4,460 & 263,365 & 18,675 \\
\hline & Congestion and emission included & 261,140 & 6,650 & 237,561 & 16,929 \\
\hline & $\%$ difference & 9.71 & -32.93 & 10.86 & 10.31 \\
\hline
\end{tabular}

Table 2. Consideration of traffic congestion and its impact on the total cost 
Column (a) of the table shows the total cost obtained by the two designs for each test network. Columns (b)(d) give the itemized cost components, including facility building cost, transportation cost, and $\mathrm{CO}_{2}$ emission cost generated from the transportation activities in column (c). Note that the congestion effect is included in the first design when the transportation costs in column (c) and the emission cost in column (d) are calculated. The percentage differences of the total cost and the itemized cost components between the two designs are shown in every third row of each test network. Column (b) shows that the proposed approach always builds more facilities than the first design, thus inducing higher facility building costs. This is intuitive, since the developed approach decides to construct an additional facility once the reduction in the sum of transportation and emission costs - due to re-assigning facility users to the new facility - becomes larger than the building cost of the given facility. Columns (c)

a)

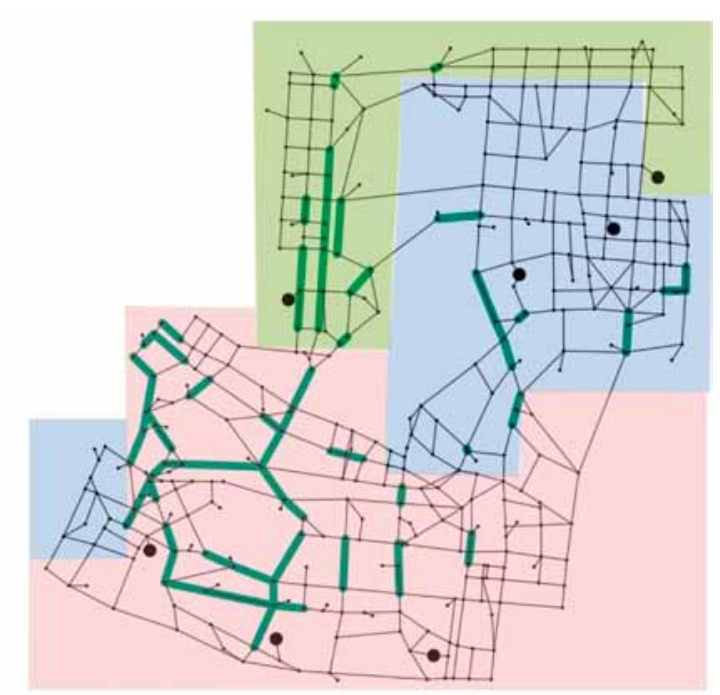

b)

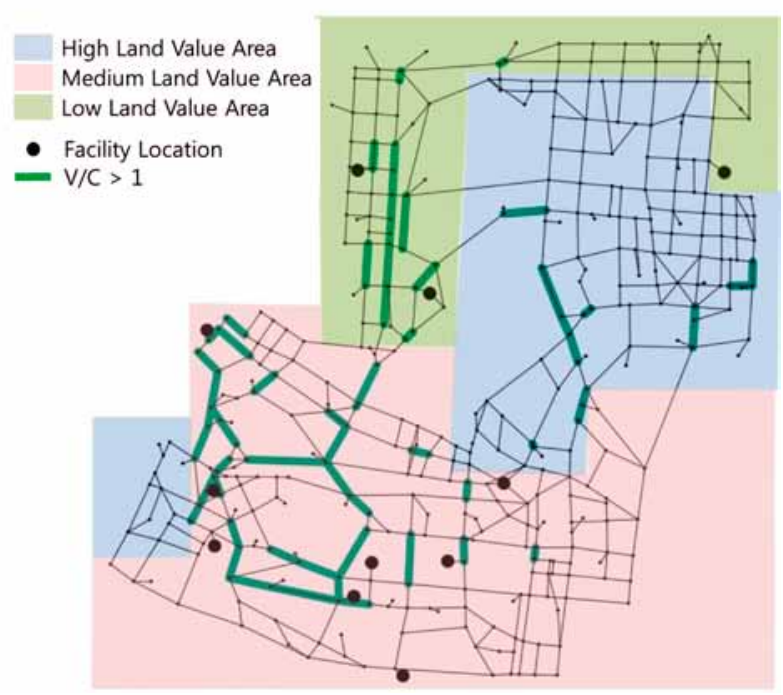

Fig. 3. Facility-location plans for Incheon network (a) ignoring traffic congestion and (b) with considering traffic congestion and emission cost in Incheon network (KTDB 2014) and (d) demonstrate that the second design generated less transportation and emission costs compared to the first, across all examples. Overall, the total cost in column (a) shows that the proposed approach has the ability to find better solutions than the first design, with significant benefits in the large-scale network. It should be noted that the heuristic solution of the developed model is better than the optimal or near-optimal solutions of the conventional problem.

Figure 3 and Table 3 show a detailed comparison of Incheon network case study with and without considering traffic congestion. Here, case (b) represents a congestion pattern of network when the plan of facility ignores the traffic congestion and the conventional fixed transportation cost method is applied. The result indicates that case (b) causes additional congestion on overall network, which leads to more than $10 \%$ increase of transportation and emission cost, and noticeably, 7 number of links and $3.27 \mathrm{~km}$ length of roadway have reached volume/capacity $(\mathrm{V} / \mathrm{C})$ ratio greater than 1 , mostly west and south part of the city.

Table 3. Comparison of cases of ignoring traffic congestion and considering traffic congestion and emission cost in Incheon network

\begin{tabular}{cccc}
\hline \multirow{2}{*}{ Network } & Design & $\begin{array}{c}\text { Number of } \\
\text { link with } \\
V / C>1\end{array}$ & $\begin{array}{c}\text { Total length } \\
\text { of link with } \\
V / C>1\end{array}$ \\
\hline \multirow{2}{*}{ Incheon } & Ignoring congestion & 125 & 53.28 \\
\cline { 2 - 4 } & $\begin{array}{l}\text { Congestion and } \\
\text { emission included }\end{array}$ & 118 & 50.01 \\
\hline
\end{tabular}

To explore how consideration of the $\mathrm{CO}_{2}$ emission affects the number and locations of facilities as well as the traffic demand routing, three scenarios with different $\mathrm{CO}_{2}$ emission factors were tested in the Incheon network: (i) a benchmark model with no consideration of the $\mathrm{CO}_{2}$ emission cost (i.e., $\beta=0 \$ / \mathrm{tCO}_{2}$ ), (ii) the proposed model in which $\mathrm{CO}_{2}$ emission cost was incorporated into the objective function (i.e., $\beta=150 \$ / \mathrm{tCO}_{2}$ ), and (iii) a high emission cost model for the case that a dramatic increase in the $\mathrm{CO}_{2}$ emission factor occurs (i.e., $\beta=900 \$ / \mathrm{tCO}_{2}$ ); the $\mathrm{CO} 2$ emission factor applied in this scenario has been estimated by many studies (Ackerman, Stanton 2012; Babonneau et al. 2014; Anandarajah et al. 2013) reflecting future uncertainty, for example, severe environmental problems (e.g., global warming) and the implementation of strict environmental policies (e.g., increased carbon tax). The results are summarized in Table 4.

The operational cost is given in column (a) of Table 4, representing the sum of the facility building cost in column (b) and the transportation cost in column (c). Note that the operational cost gradually increases as the $\mathrm{CO}_{2}$ emission factor increases, and that column (b) includes 
information on the number of facilities constructed in each scenario. In general, the number of facilities as well as their building cost increase as the emission factor grows since reduction in the sum of transportation and emission costs dominates the cost of building additional facilities. While the number of facilities in the proposed model and the high emission cost model are the same, the total building costs are different since the high emission cost model chooses more expensive candidate locations (e.g., central business districts) in order to reduce the emission cost. Column (d) describes the $\mathrm{CO}_{2}$ emission cost associated with each scenario. Note that the total emission costs in the benchmark model and the high emission cost model were recalculated based on the decisions made in each scenario, but $\beta=150 \$ / \mathrm{tCO}_{2}$ was applied for normalization, which was included in the parentheses in column (d). It can be seen that the normalized emission cost decreases as the $\mathrm{CO}_{2}$ emission factor increases. Although the difference in emission cost is small compared to the total cost, this causes an alteration in the number and locations of facilities.

Figures 4(a)-(c) illustrate where the facilities would be built in the Incheon network based on the results shown in Table 4. Note that the study area was categorized into three groups (i.e., high, medium, and low land value areas), as shown in the legend. Thick green lines represent heavily congested network links where the ratio of assigned traffic volume to the roadway capacity is greater than one. One can observe that facilities are densely located near the heavily congested regions in all figures. Figure 4(a) illustrates the nine community centers constructed with the benchmark model, while Figures 4(b) and (c) show a total of 11 community centers which are constructed in both the proposed model and the high emission cost model, with obvious differences in location. As the number of facilities increases in a congested area, we can expect to benefit from a decrease in the sum of transportation costs and emission costs. Thus, Figures 4 (b) and (c) show less congested patterns in the network compared to those in Figure 4(a). Note that the proposed model chose to construct eight facilities in the medium land price area and three facilities in low-priced area, while the high emission cost model resulted in one facility in the high land price area, seven in the medium-priced area, and three in the low-priced area. This trend explicitly indicates a)

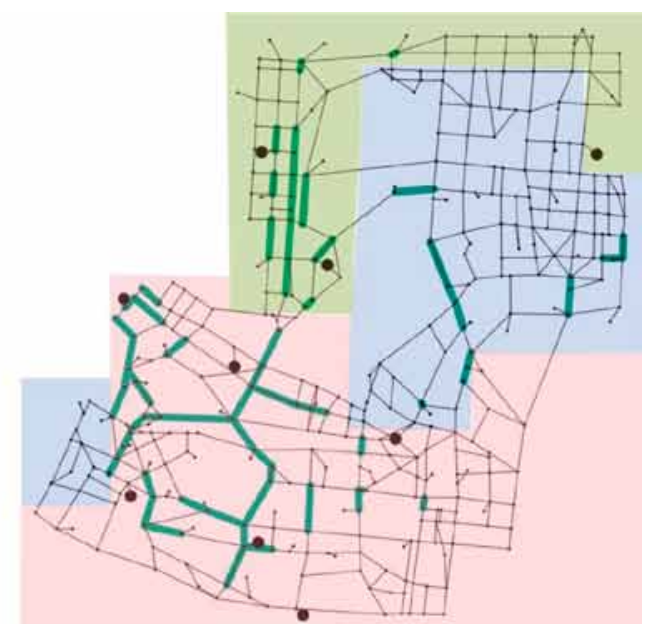

b)

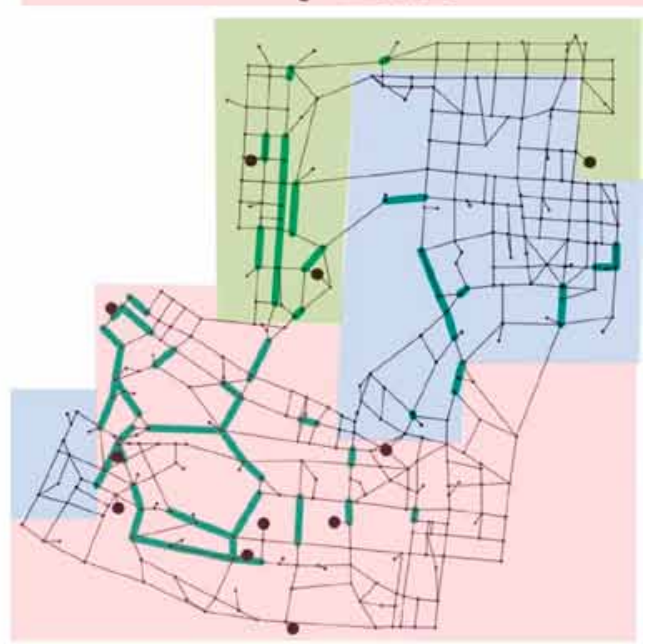

c)

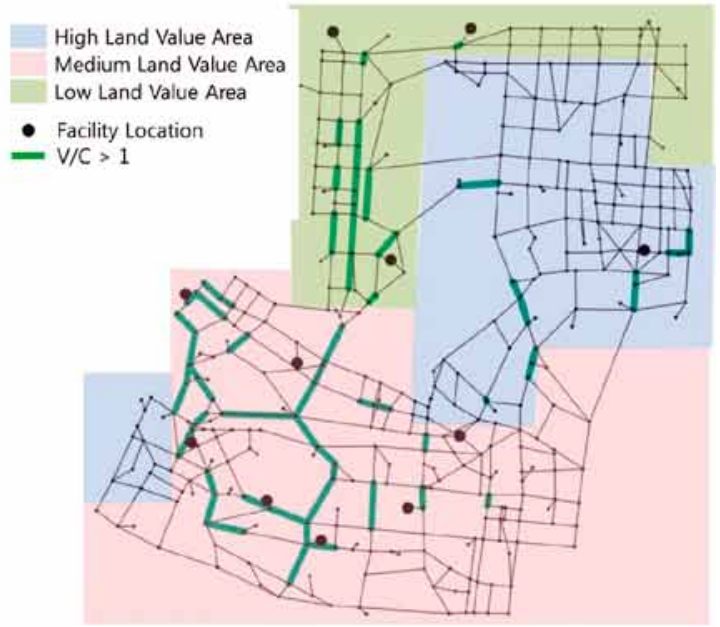

Fig. 4. Facility-location plans for three emission factor scenarios, benchmark model (a), proposed model (b), and high emission cost model (c) in Incheon network (KTDB 2014)

Table 4. Impact of $\mathrm{CO}_{2}$ emission consideration on the solution for the Incheon network

\begin{tabular}{lcccc}
\hline \multicolumn{1}{c}{ Scenario } & $\begin{array}{c}\text { (a) Operational } \\
\text { cost in } \$: \\
\text { (b) }+(\mathrm{c})\end{array}$ & $\begin{array}{c}\text { (b) Facility cost in } \\
\text { \$ (\# of facilities) }\end{array}$ & $\begin{array}{c}\text { (c) Transportation } \\
\text { cost in } \$\end{array}$ & $\begin{array}{c}(\mathrm{d}) \mathrm{CO}_{2} \text { emission cost in } \$ \\
\left(\beta=150 \$ / t \mathrm{CO}_{2}\right)\end{array}$ \\
\hline Benchmark model $\beta=0$ & 244,124 & $5,370(9)$ & 238,754 & $0(17,021)$ \\
Proposed model $\beta=150$ & 244,211 & $6,650(11)$ & 237,561 & $16,929(16,929)$ \\
High emission cost model $\beta=900$ & 244,465 & $6,770(11)$ & 237,695 & $101,275(16,879)$ \\
\hline
\end{tabular}


that as the $\mathrm{CO}_{2}$ emission factor becomes significant, the suggested model tries to reduce the emission costs despite an increase in the facility building costs.

In summary, it was shown through various network examples that a tabu search generates the best solution, followed by a memetic algorithm, and a conventional genetic algorithm performs worse than other algorithms developed in this study. Also, a series of case studies show that the proposed modeling framework builds more facilities, and thus induces more facility building cost than the conventional fixed-charge facility location model. However, as the number of constructed facility increases in congested areas, we can eventually expect benefit from decrease in the sum of transportation cost and $\mathrm{CO}_{2}$ emission cost; this trend was explicitly shown when the $\mathrm{CO}_{2}$ emission factor becomes significantly large. Thus, the proposed methodology will be able to contribute to achieve environmental-friendly transportation systems by considering traffic congestion and the subsequent vehicle emission.

\section{Conclusions and future study}

The construction of new facilities increases the traffic demand around them, which may adversely affect the roadway traffic conditions or level of service. In most urban areas where the existing traffic is at or near capacity, the effects of constructing new facilities on network congestion are more significant. Consequently, the congestion effects should be considered when deciding the locations of facilities. In this study, the transportation costs caused by both facility users and existing general roadway users were incorporated into the conventional facility-location problem. Furthermore, the $\mathrm{CO}_{2}$ emission costs caused by all types of traffic (i.e., re-routing of general roadway users as well as assignment of facility users) were taken into account. The proposed comprehensive modeling framework provides insight on the bidirectional relationship between the classical facility location model and the traffic assignment model, with consideration of the environmental impacts of vehicle movements. Facility location decisions and network congestion patterns are iteratively updated in the model until the system-wide total cost converges to a near-optimum value. Since both link travel cost and link specific $\mathrm{CO}_{2}$ emission factor are formulated as nonlinear functions with respect to the link traffic volume (rather than a fixed value), heuristic approaches including tabu search and memetic algorithms were proposed to solve the problem. The proposed methods and a conventional genetic algorithm were applied to three test examples ranging from small to large-scale transportation networks to compare the performances of different algorithms. The results indicated that tabu search provides the best solution among the algorithms considered. The selected algorithm was then applied to locate community service centers in
Incheon City under multiple scenarios. The proposed model was compared with both the conventional facility location model and the high emission cost model to investigate the impacts of traffic congestion and the subsequent $\mathrm{CO}_{2}$ emissions on the real-world large size network. The proposed approach demonstrated significant reduction of both the transportation and GHG emission costs compared to the conventional facility-location model, thus generating the lowest system-wide cost. The results in this study will be useful for enhancing public benefit and social welfare by reducing the adverse impacts from traffic congestion and the related vehicle emissions in congested urban areas.

Future research is suggested as follows. First, the traffic demand considered in this study, which included background traffic and facility demand traffic, were converted into passenger car volume, which is a conventional traffic demand assignment procedure. However, this may cause a bias in calculating the total $\mathrm{CO}_{2}$ emission cost from various vehicle types. This limitation can be resolved if the background traffic and facility demand traffic data include information on the shares of different vehicle types, such as passenger cars, SUVs, buses, and trucks. Second, this study assumed that all cost components are associated with fixed unit cost. This simplification could be relaxed by considering changes in the unit price of each component over time, resulting in more accurate facility-location decisions. Our model could be further developed into a multi-period facility-location problem by considering time-frames.

\section{Disclosure statement}

We do not have any competing financial, professional, or personal interests from other parties.

\section{Acknowledgments}

The authors thank the anonymous reviewers for their insightful comments.

\section{Funding}

This work was supported by the National Research Foundation of Korea [grant number 2015R1C1A1A02037285]; and INHA UNIVERSITY Research Grant [grant number INHA-51368].

\section{References}

Ackerman, F; Stanton, E. A. 2012. Climate risks and carbon prices: revising the social cost of carbon, Economics 6(10): 1-25. http://dx.doi.org/10.5018/economics-ejournal.ja.2012-10

Aikens, C. H. 1985. Facility location models for distribution planning, European Journal of Operational Research 22(3): 263-279. http://dx.doi.org/10.1016/0377-2217(85)90246-2 
Air Resource Board of California Environmental Protection Agency. 2014. Reducing air pollution - ARB programs: mobile sources [online], [cited 12 May 2014]. Available from Internet: http://www.arb.ca.gov/msprog/msprog.htm

Aksen, D.; Aras, N. 2012. A bilevel fixed charge location model for facilities under imminent attack, Computers and Operations Research 39(7): 1364-1381.

http://dx.doi.org/10.1016/j.cor.2011.08.006

Anandarajah, G.; Dessens, O.; McGlade, C. 2013. Modelling of global energy scenarios under $\mathrm{CO} 2$ emissions pathways with TIAM-UCL. UCL Energy Institute, London, UK.

API. 1998. Impacts of market-based greenhouse gas emission reduction policies on U.S. manufacturing competitiveness. American Petroleum Institute.

Al-Sultan, K. S.; Al-Fawzan, M. A. 1999. A tabu search approach to the uncapacitated facility location problem, Annals of $\mathrm{Op}$ erations Research 86: 91-103.

http://dx.doi.org/10.1023/A:1018956213524

Arostegui Jr., M. A.; Kadipasaoglu, S. N.; Khumawala, B. M. 2006. An empirical comparison of tabu search, simulated annealing, and genetic algorithms for facilities location problems, International Journal of Production Economics 103(2): 742-754. http://dx.doi.org/10.1016/j.ijpe.2005.08.010

Babonneau, F. L. F.; Haurie A.; and Vielle, M. 2014. A stochastic dynamic game of the 2050 European Energy Roadmap with CCS, in 14th IAEE European Energy Conference, 28-31 October 2014, Roma, Italy.

Bai, Y.; Hwang, T.; Kang, S.; Ouyang, Y. 2011. Biofuel refinery location and supply chain planning under traffic congestion, Transportation Research Part B: Methodological 45(1): 162175. http://dx.doi.org/10.1016/j.trb.2010.04.006

Bar-Gera, H. 2009. Transportation network test problems [online], [cited 25 March 2014]. Available from Internet: https:// github.com/bstabler/TransportationNetworks

Beasley, J. E. 1993. Lagrangean heuristics for location problems, European Journal of Operational Research 65(3): 383-399. http://dx.doi.org/10.1016/0377-2217(93)90118-7

Brownlee, J. 2013. Clever algorithms: nature-inspired programming recipes [online], [cited 11 March 2014]. Available from Internet: http://www.cleveralgorithms.com/

Cornuejols, G.; Sridharan, R.; Thizy, J. M. 1991. A comparison of heuristics and relaxations for the capacitated plant location problem, European Journal of Operational Research 50(3): 280-297. http://dx.doi.org/10.1016/0377-2217(91)90261-S

Daskin, M. S. 1995. Network and discrete location: model, algorithms, and applications. New York: John Wiley and Sons. http://dx.doi.org/10.1002/9781118032343

Davidson, K. B. 1966. A flow-travel time relationship for use in transportation planning, Proceedings of $3 r d$ ARRB Conference 3(1): 183-194.

Diabat, A.; Simchi-Levi, D. 2009. A carbon-capped supply chain network problem, Proceedings of IEEE International Conference on Industrial Engineering and Engineering Management, 8-11 December 2009, IEEE, 532-527.

http://dx.doi.org/10.1109/IEEM.2009.5373289

Erlenkotter, D. 1978. A dual-based procedure for uncapacitated facility location, Operation Research 26(6): 992-1009. http://dx.doi.org/10.1287/opre.26.6.992

Figliozzi, M. 2011a. The impacts of congestion on time-definitive urban freight distribution networks $\mathrm{CO}_{2}$ emission levels: results from a case study in Portland, Oregon, Transportation Research Part C: Emerging Technologies 19(5): 766-778. http://dx.doi.org/10.1016/j.trc.2010.11.002

Figliozzi, M. 2011b. Vehicle routing problem for emissions minimization, Transportation Research Record: Journal of the Transportation Research Board 2197(2): 1-7. http://dx.doi.org/10.3141/2252-01

Goldberg, D. E. 1989. Genetic algorithms in search, optimization, and machine learning. New York: Addison-Wesley.

Hajibabai, L.; Ouyang, Y. 2013. Integrated planning of supply chain networks and multimodal transportation infrastructure expansion: model development and application to the biofuel industry, Computer-Aided Civil and Infrastructure Engineering 28(4): 247-259. http://dx.doi.org/10.1111/j.1467-8667.2012.00791.x

Hajibabai, L.; Bai, Y.; Ouyang, Y. 2014. Joint optimization of freight facility location and pavement infrastructure rehabilitation under network traffic equilibrium, Transportation Research Part B: Methodological 63: 38-52. http://dx.doi.org/10.1016/j.trb.2014.02.003

Harris, I.; Mumford, C.; Naim, M. 2009. The multi-objective uncapacitated facility location problem for green logistics, Proceedings of IEEE Congress on Evolutionary Computation, 18-21 May 2009, Trondheim, Norwa, 2732-2739. http://dx.doi.org/10.1109/cec.2009.4983285

Harris, I.; Mumford, C. L.; Naim, M. 2011. An evolutionary biobjective approach to the capacitated facility location problem with cost and $\mathrm{CO}_{2}$ emissions, Proceedings of the 13th Annual Conference on Genetic and Evolutionary Computation, 12-16 July 2011, Dublin, Ireland, 697-704.

Hickman, A. J. 1999. Methodology for calculating transport emissions and energy consumption. Transport Research Laboratory, Crowthorne, UK.

Jaccard, M. 2005. Hybrid energy-economy models and endogeneous technological change, in R. Loulou, J.-P. Waaub, G. Zaccour (Eds.). Energy and environment. New York, NY: Springer. http://dx.doi.org/10.1007/0-387-25352-1_4

Jang, M.; Kim, J. A.; Sun, S. T. 2010. Development and evaluation of laws and regulation for the low-carbon and green growth in Korea, International Journal of Urban Sciences 14(2): 191206. http://dx.doi.org/10.1080/12265934.2010.9693676

Jaramillo, J. H.; Bhadury, J.; Batta, R. 2002. On the use of genetic algorithms to solve location problems, Computers \& Operations Research 29(6): 761-779. http://dx.doi.org/10.1016/S0305-0548(01)00021-1

Kim, S. 2012. Intermodal freight network design problem with GHG emission constraints: PhD dissertation. Seoul National University, Seoul, Republic of Korea.

KTDB. 2014. Korea Transport Database [online], [cited 21 April 2014]. Available from Internet: http://www.ktdb.go.kr

Lee, J. K.; Lee, S. 2010. Reviewing Korea's strategic plan of adaptation and mitigation for sustainable development and climate change, International Journal of Urban Sciences 14(1): 60-72. http://dx.doi.org/10.1080/12265934.2010.9693664

Lim, M.; Daskin, M. S.; Bassamboo, A.; Chopra, S. 2010. A facility reliability problem: formulation, properties, and algorithm, Naval Research Logistics 57(1): 58-70.

MLTM. 2011. Evaluation guideline for transportation facility investment. Ministry of Land, Transportation and Maritime, Seoul, Republic of Korea. 
Nozick, L. K. 2001. The fixed charge facility location problem with coverage restrictions, Transportation Research Part E: Logistics and Transportation Review 37(4): 281-297. http://dx.doi.org/10.1016/S1366-5545(00)00018-1

NRT. 2012. Reality check: the state of climate progress in Canada. National Roundtable on the Environment and the Economy, Ottawa, Canada.

Parry, I. W. H.; Toman, M. A. 2001. Greenhouse gas "early reduction" programs: a critical appraisal, in M. A. Toman (Ed.). Climate change economics and policy: an RFF anthology. Washington, DC: RFF Press.

Pirkul, H.; Jayaraman, V. 1998. A multi-commodity, multi-plant, capacitated facility location problem: formulation and efficient heuristic solution, Computers and Operations Research 25(10): 869-878.

http://dx.doi.org/10.1016/S0305-0548(97)00096-8

Prins, C.; Prodhon, C.; Ruiz, A.; Soriano, P.; Calvo, R. W. 2007. Solving the capacitated location-routing problem by a cooperative lagrangean relaxation-granular tabu search heuristic, Transportation Science 21(4): 470-483.

http://dx.doi.org/10.1287/trsc.1060.0187

Raj, K. A. A. D.; Rajendran, C. 2012. A genetic algorithm for solving the fixed-charge transportation model: two-stage problem, Computers \& Operations Research 39(9): 20162032. http://dx.doi.org/10.1016/j.cor.2011.09.020

RSCEP. 2010. Environmental and health impacts of Canada's Oil Sands Industry. The Royal Society of Canada Expert Panel, Ottawa, Canada.

Sheffi, Y. 1985. Urban transportation networks: equilibrium analysis with mathematical programming methods. Englewood Cliffs, NJ: Prentice Hall.

Shukla, A.; Alam, M. 2010. Assessment of real world on-road vehicle emissions under dynamic urban traffic conditions in Delhi, International Journal of Urban Sciences 14(2): 207-220. http://dx.doi.org/10.1080/12265934.2010.9693677

Sörensen, K.; Vanovermeire, C.; Busschaert, S. 2012. Efficient metaheuristics to solve the intermodal terminal location problem, Computers \& Operations Research 39(9): 20792090. http://dx.doi.org/10.1016/j.cor.2011.10.005

Sridharan, R. 1995. The capacitated plant location problem, European Journal of Operational Research 87(2): 203-213. http://dx.doi.org/10.1016/0377-2217(95)00042-O

Srivastava, S. K. 2007. Green supply-chain management: a stateof-the-art literature review, International Journal of Management Reviews 9(1): 53-80. http://dx.doi.org/10.1111/j.1468-2370.2007.00202.x

Statistics Korea. 2014. Transportation sector social cost [online], [cited 30 March 2014]. Available from Internet: http://index. go.kr/smart/chart_view.jsp?idx_cd=2971

Tragantalerngsak, S.; Holt, J.; Rönnqvist, M. 1997. Lagrangian heuristics for the two-echelon, single-source, capacitated facility location problem, European Journal of Operational Research 102(3): 611-625.

http://dx.doi.org/10.1016/S0377-2217(96)00227-5

TRB. 2000. Highway capacity manual. Transportation Research Board, Washington, DC.

U.S. EPA. 2013. Fast facts: U.S. transportation sector greenhouse gas emissions 1990-2011 [online], [cited 11 February 2014]. Available from Internet: http://www.epa.gov/otaq/climate/ documents/420f13033a.pdf

Venables, H.; Moscardini, A. 2006. An adaptive search heuristic for the capacitated fixed charge location problem, Proceedings of Ant Colony Optimization and Swarm Intelligence, 4-7 September 2006, Brussels, Belgium, 348-355.

Wang, F.; Lai, X.; Shi, N. 2011. A multi-objective optimization for green supply chain network design, Decision Support Systems 51(2): 262-269. http://dx.doi.org/10.1016/j.dss.2010.11.020

Wardrop, J. G. 1952. Road paper No. 36: Some theoretical aspects of road traffic research, ICE Proceedings: Engineering Divisions 1(3): 325-362.

Xie, F.; Jia, R. 2012. Nonlinear fixed charge transportation problem by minimum cost flow-based genetic algorithm, Computers \& Industrial Engineering 63(4): 763-778. http://dx.doi.org/10.1016/j.cie.2012.04.016

Taesung HWANG. PhD. Assistant Professor in Asia Pacific School of Logistics and Graduate School of Logistics at Inha University, Incheon, Republic of Korea. Bachelor of Science in Civil and Environmental Engineering from Seoul National University, 2003; Master of Science in Civil and Environmental Engineering from the University of California at Berkeley, 2007; and Ph.D. in Civil Engineering from the University of Illinois at Urbana-Champaign, 2014. Research interest lies in freight shipment modeling, logistics systems planning, and sustainability and environmental impacts of transportation.

Minho LEE. Bachelor of Science in Civil and Environmental Engineering from Korea University, in 2011, and Master of Science in Civil and Environmental Engineering (Transportation Engineering Program) from Korea University, in 2013. Research interests include logistics systems planning, traffic flow theory, and software engineering.

Chungwon LEE. PhD. Associate Professor in the Department of Civil and Environmental Engineering at Seoul National University, Seoul, Republic of Korea. Both Bachelor of Science (1986) and Master of Science (1988) in Civil and Environmental Engineering from Seoul National University; and Doctoral degree in Civil and Environmental Engineering from the University of Texas at Austin in 1998. Research interest lies in multi-modal public transportation planning and supply chain management.

Seungmo KANG. PhD. Associate Professor in the School of Civil, Environmental and Architectural Engineering at Korea University, Seoul, Republic of Korea. Both Bachelor of Science (1998) and Master of Science (2000) in Civil and Environmental Engineering from Seoul National University; and Doctoral degree (2008) in Civil Engineering from the University of Illinois at Urbana-Champaign. Research interest includes transportation network modeling and supply chain management, transportation operations and safety. 